\title{
Human subjects in clinical trials: Ethical considerations and concerns
}

\author{
Ahmad W'* Moeen Al-Sayed ${ }^{2 *}$ \\ ${ }^{1}$ Advanced clinical specialist- Medical Genetics, King Faisal Specialist Hospital \& Research Center, Riyadh:11211 - MBC:75, Saudi Arabia \\ ${ }^{2}$ Professor of Genetics, College of Medicine, Alfaisal University, Department of Medical Genetics, King Faisal Specialist Hospital and Research Center, Saudi Arabia
}

\section{Introduction}

Clinical research is a lengthy and costly process. Subject recruitment and retention is an essential step to help lowering the cost and the length of clinical trials [1]. Good quality research is crucial for determining the clinical and cost effectiveness of health care systems, at the same time recruitment of sufficient participants is a cornerstone for good quality research that tests hypotheses with confidence and minimizes bias. There are different methods for enhancing recruitment rates; in this paper, I am going to discuss offering incentives for potential subjects and staffs who help in recruiting research subjects, particularly principal investigator [2]. Also, I am going to highlight some ethical concerns and considerations that related to recruiting human subjects in clinical research. Recruitment and retention of research subjects is crucial for medical advancement and providing data that contribute in directing practice and policy [3]. Informed consent is a critical element in subject recruitment process and it is a crucial step for conducting an ethical research. Good Clinical Practice (GCP) guidelines and International Conference on Harmonization (ICH) recommendations are fundamental for writing the informed consent. Understanding the concept of vulnerable subjects is another area clinical research personnel need to focus on and understand [1].

\section{Human subjects in clinical trials: Ethical consider- ations and concerns}

Good Clinical Practice (GCP) is international guideline to ensure that clinical trials are designed, conducted, implemented, monitored, audited, recorded, analyzed, and reported scientifically and ethically. It also aims to protect human subject rights, integrity, and confidentiality. In 1997 GCP became effective, though not legally acknowledged at that point. In 2004, in the United Kingdom/Europe GCP became a legal obligation for all clinical trials involving investigational products. Historically, unethical and horrific research took place during the World War II by German clinicians at Nazi war cantonment, which led to the establishment of Nuremberg Code in 1947 [4]. The Universal Declaration of Human Rights became effective by the United Nation as a result of inhuman trials conducted during the World War II. In 1964, the Declaration of Helsinki was emerged and adopted by the World Medical Association and served as a backbone for the ethical principles that formulated the current International Conference on Harmonization (ICH)-GCP guidelines. In 1962, the Kefauver-Harris Amendments were adopted and required the FDA to assess all new drugs for safety and efficacy as a result of thalidomide tragedy that led to the born of 10,000 infants with fetal limb deformities in more than 20 countries worldwide [4].

Different organizations and committees worldwide adopted several guidelines and documents in this regard. Thus, a need for consolidating all these guidelines into one global document appeared. To achieve this goal, in 1996, the ICH Guideline was issued by the International Conference for Harmonization of Technical Requirements for Registration of Pharmaceuticals for Human Use (ICH) [4]. Representatives of regulatory bodies and pharmaceutical companies from Japan, United States, European Union, Australia, Canada, and Nordic Countries as well World Health Organization (WHO) participated in the development of these guidelines. In January1997, the guideline became effective and implemented for clinical trials involving human subjects [4].

According to the ICH-GCP guidelines all clinical trials should be conducted in compliance with ethical standards, clear scientific proof, and benefit overweigh risk; and a clear well-documented protocol is required. Obtaining an informed consent and affirming confidentiality. The trial staff should receive adequate training along with their appropriate qualifications. Data should be documented accurately and easily accessible and available. Manufacturing the investigational products should be in accordance with Good Manufacturing Practice (GMP) guidelines [4].

\section{Informed consent process}

The process of informed consent is tailored to inform the subject of rights, risks, and benefits when participating in a clinical trial. Informed consent is an essential element for conducting an ethical research that involves human subjects. As an outcome of the 1974 National Research Act Belmont Report was issued in 1979 in the United States. This report serves as guidance for biomedical and behavioral researchers to find a summary of the basic ethical principles to protect human subjects basically: beneficence, justice, and respect for individuals. The process of informed consent is crucial in achieving these principles. However, to further protect human subjects, the informed consent process involves the verbal discussion with the possible subject along with the paper document. The Principal Investigator is accountable to explain the informed consent and determine that the subject has understood the information clearly and given his/her voluntary approval for participating in a particular trial [5].

When obtaining the informed consent from the subject or their legally guardian, the possibility of coercion or undue influence must be minimized. The informed consent document (ICD) should contain

Correspondence to: Wijdan Ahmad, Advanced clinical specialist- Medical Genetics, King Faisal Specialist Hospital \& Research Center, Riyadh:11211 MBC:75, Saudi Arabia, E-mail: wahmad@kfshrc.edu.sa

Received: April 01, 2018; Accepted: April 11, 2018; Published: April 13, 2018 
information in an understandable language to the subject, thus ICD, advertisements, and other study-associated documents may require translation as well, if necessary, having a person on site to answer queries. The ICD language should not seem to disregard or ignore any subject rights or exempt any of the clinical research stakeholders from liability for negligence. The aim, the expected length of the study, and all required procedures the subject will go through should be stated clearly in the ICD. In addition, ICD should describe the risks, the benefits of the research for subjects or others, other treatment options, and confidentiality of subjects' data [5].

In research that may lead to minimal risk for future subjects, regulatory bodies acquire explanation of whether medical care or compensation will be provided in case of injury. Beside their right of free participation in research, subjects have the right to know that they are free to withdraw from the trial at any given point [5]. Furthermore, the Food and Drug Administration (FDA) view advertising as the initial phase in the informed consent process. Therefore, the advertising contents should be in harmony with data that is existed in the approved ICD. The advertisement should not promise providing free treatment or claim the safety or effectiveness of the investigational or experimental drug, device, or biologic [5].

The Institutional review board (IRB) is responsible to identify the degree of risk in a clinical trial. Before study approval, IRB must determine the risks and benefits for a child subject in a research that involves children and to ensure its compliance with regulatory guidelines. Whenever indicated, the IRB must assess the potential risk/ benefit and decide the importance of the presence of a legal guardian and the assent (the child agreement to participate in the study) of the child accordingly. Also, the investigator must explain the alternative treatment options and the risk/ benefit of the investigational treatment for the critically or seriously ill subjects. In some cases where the subject is very sick or too sedated the IRB may approve the use of a "short form" of the ICD. However, if any subject is unable to understand the provided information in the ICD, the IRB may mandate the utilization of a legally authorized representative [5].

Usually, the notion of the modern informed consent is questionable considering the difficulty of determining what is an acceptable and sufficient level of information that make the consent to be valid. Generally, consent can be valid when it includes the understanding of voluntary participation, purpose of the research, risk and benefit ratio, and the procedures that may be required throughout the study. It is difficult to establish the adequate level of information at each level of these stages. In medical research, the primary end of research is not to focus on the direct benefit of research subjects, despite the possibility of subjects to receive a therapeutic benefit [6] Some argue that the current trend concerns the issue of paternalism, for instance, there is a concern regarding level of protection stated in the present guidelines that may interferes with the autonomous choices of participants. A patient may like to take higher risk to get better benefit, for example, taking part in an innovative promising therapy. Conversely, an end stage patient may wish to participate in research for the sake of benefiting others or future generations. Current guidelines are inconsistent with this view as they put a heavy emphasis on protection of participants [6].

\section{Subject recruitment}

"Recruitment refers to the process of selection- from notification of the study to the enrolment of participants. It starts with communication between the researcher and the potential participants and aims to recruit appropriate participants who are representative of the target population" [7]. Many researchers have highlighted the issue of insufficient recruitment. On the other hand, the process of keeping subjects in the study known as retention. Lasagna's law or the 'funnel effect' is a phenomenon that refers to researchers' tendency to aggravate the number of potential patients who meet the inclusion criteria and would be willing to participate in a specific clinical trial [7]. "Evidence indicates that only $10 \%$ of subjects survive the' recruitment funnel." [7].

Recruitment and retention of research subjects is crucial for the progress of medical advancement and to provide data that help in guiding practice and policy. According to Dickert et al. the recruitment for reduced cardiac ejection fraction trial to assess the use of Warfarin versus Aspirin took more than seven years. Whereas, a successful randomization of 7141 subjects in less than three years was achieved for a study that intended to prove the clinical effectiveness of Nesiritidein decompensated heart failure [3]. The specific rational for the variation in recruitment is not clear and differ from site to site and study to another. Unfortunately, clinical knowledge may be negatively impacted by slow recruitment and insufficient retention; variability in subject's attributes and withdrawal rates may lead to restriction of the generalizability of the study outcome [3].

Furthermore, poor recruitment consumes the resources, increases the cost, and may lead to moving research outside United States. "According to a recent Institute of Medicine-National Cancer Institute report, $40 \%$ of National Cancer Institute-funded trials do not complete enrollment" [3]. To face these challenges that may hinder the advancement of health care, there have been suggestions for relaxing the regulatory requirements, especially for low-risk comparative effectiveness of available treatments. Thus, the need for examining and evaluating different methods for maximizing subject recruitment and retention is obvious [3].

\section{Payment for human subjects}

In the United States, payment for human subjects is a deep-rooted and a well-known practice that has been reported for more than 100 years ago. For example, in 1820, the father of gastric physiology; William Beaumont offered his patient food, shelter, clothing, and \$150 to examine his stomach contents for one year as he was suffering of incompletely healed gunshot wound to his stomach [8]. In 1920s and 1930s, payment for human subjects and other monetary compensation, such as food and transportation became a regular and routine practice. In 1950s, the NIH Clinical Center opened documents that reported payment for healthy subjects for their participation in medical research or money was provided to those who recruited the research subjects especially the church. Currently, in the newspaper and websites advertisements, it is common to see this statement "financial compensation provided." Despite of being common, the practice of paying subjects continues to be an issue [8]. Some considers paying subjects as coercion where as others believe it is an essential method for a successful recruitment process; others see payment as fair and appropriate, especially for healthy subjects. The issue of payment of research subjects is not addressed clearly in the US Code of Federal Regulations governing clinical research [8]. Investigators are left with unclear and minimal guidance in this regard. The responsibility of ensuring that the proposed amount and schedule of payment is fair and adequate falls on the Institutional review boards (IRBs); however, these regulatory bodies operate with minimal and broad guidelines that make their task very difficult. Thus, in the US payment varies from state to state and from study to another [8]. Payment enhances subjects to participate in clinical trials. Many studies have reported that 
payment has increased response rate to written surveys, but when it comes to clinical trials, there is less known of the effect of payment in recruitment rate [8]. There are different ways in which payment might increase recruitment. First, offering money as reimbursement for time or expenses might encourage subjects to participate so they will not refrain from participation because they do not afford losing their own money. On the other hand, payment could enhance recruitment of individuals who believe in being fairly compensated for their time and effort. Besides enhancing recruitment, money may help to achieve other goals, such as racial, gender, ethnic and social diversity of subject pool [8].

Moreover, there is limited evidence that supports the necessity of payment for improving subject recruitment in clinical research. Other reasons may encourage individuals to participate in clinical research. For example, healthy volunteers may participate for curiosity, altruism, sensation seeking, and desire to help others and take part in health advancement. Patient-subjects may participate hoping they will benefit out of this new therapy. With limited evidence of how much money may encourage individuals to participate in clinical research, it is hard to recognize the real effect of payment in helping to accomplish recruitment goals [8].

Some argue that money may impair judgment or jeopardize voluntary decision making. However, making decisions is usually a multifactorial and complicated process and it is rarely based only on money, as I have mentioned earlier people may participate in clinical research for many different reasons other than money. "In one study, most respondents (75\%) thought an offer of $\$ 500$ for research participation could impair the judgment of others, but many fewer (20\%) thought it would impair their own judgment" [8]. Unlike healthy subjects, there are more ethical concerns regarding payment of patent subjects because they are considered vulnerable. Once again, if healthy subjects are being paid for their time and effort thus patient subject have the same right equally of receiving payment. In addition, paying patient subjects may help them to not feel obligated to participate in a clinical trial and say no for their physician [8].

Frequently, subjects are being offered incentives to encourage their participation in clinical research. However, this practice usually raises ethical considerations and concerns. For instance, undue inducement, bias, and clinical research exploitation stay on the top of these ethical concerns [9]. The amount of monetary compensation depends on the research type and the required amount of time needed to accomplish research activities and procedures. For example, subjects in social or behavioral research may get $\$ 50$ whereas healthy subjects participating in phase I trial may receive hundreds or thousands of dollars [9]. The reasons behind paying subjects include enhancing recruitment process and to compensate subjects for their time, effort, and travel [9]. Studies have shown than offer of financial incentives is the main driving force that encourages individuals to participate in clinical research. Gaining access to medical treatment or contributing to the advancement of medical knowledge may encourage subjects to participate in clinical research. Furthermore, healthy subjects participating in phase I trials are usually encouraged by incentives whereas financial incentives play a minor role in encouraging patients to enroll in phase II and phase III trials. In addition, financial incentives probably play a minor role in well-developed countries compared with developing countries [9].

Conducting an ethical research requires a fair distribution of benefits and risks. So, research subjects deserve to get some benefits that compensate their time and effort and placing themselves at risk.
Another important aspect for paying research subjects is to appreciate their engagement in the study, which is considered essential to build the trust between investigators and subjects [9]. Informed consent is essential for conducting an ethical research and subjects must give their voluntarily and autonomous choice to participate in clinical research. One of the essential ethical issues regarding offering subjects' financial payment is that may lead to coercion or undue inducement by affecting their decision to participate for financial reasons. However, Resnik argues that coercion is using force or threat against someone to make him/her obey a demand or request thus offering money for subjects does not constitute any coercion [9]. Furthermore, money is a benefit not harm. If the subjects are offered a big amount of money or motivated by financial payment, due to a financial crisis; in this case, we could consider Undue inducement. While this debate may sound logical, empirical studies has shown that research subjects are most likely not unduly influenced by fair and adequate amount of money for participation. However, it is recommended to conduct more research to study the effect of incentives on the decision of subjects to whether/or not participate in clinical trials considering different social, psychological and economic factors that may affect their decision such as education, culture, sex, and ethnicity [9].

On the contrary, paying human subjects a very small amount of money may lead to exploitation. As the research sponsors, institutions, and investigators they are going to benefit from the study compared with a limited and unfair benefit for research subject's [9]. Exploitation might also happen with pediatric subjects, considering that parents might enroll their children to get the financial incentives for themselves. Enrollment bias is another aspect to be considered, as paying incentives may attract higher rates of subjects with a lower socioeconomic status [9]. The impact of this kind of bias could lead to a serious problem that jeopardizes the generalizability of research results. This may deplete the scientific and social value of clinical research. Further research is needed to assess the appropriate payment method an amount for research subjects [9].

\section{Payment for physicians}

The lack of incentives in clinical research is considered as a major potential barrier for subject recruitment. For example, physicians who refer subjects do not get any compensation, faculty members are rarely promoted for being investigators in multicenter trials, and the research staff payment is unassociated to recruitment success [3]. There are some ethical concerns for the protection of research subjects, based on historical research abuses, regarding incentives for researchers and subjects. However, some limited researches have falsified the concerns related to incentives leading to undue inducement. [3].

At some point, finders fees (a payment for referrals) used to be given to referring physician to compensate their efforts and their critical role for recruiting subjects. Concerns of conflict of interest led to their prohibition in academic centers. Since the role of research is critical for health care system advancement, there is a need for finding methods to provide incentives with minimizing conflict of interest and maximizing preferable outcomes [3]. Proper disclosure of incentive planning and studying patients' feedback is crucial for assessing their ethical impact. Dickert et al. believe that a randomized trial should be conducted in different sites and different geographical locations to assess the effect of incentives for clinicians. They suggest that offering incentive for clinicians based on enrollment rate, referral rate, or no payment at all. Accordingly, the outcomes and effectiveness could be measured the successful enrolment rates, subject's satisfaction and trust 
for their clinician, and comparing the cost of the trial in the different participating sites in the study [3]. Although coordinators play an important role in recruiting and retaining subjects, the incentive is not a big concern relates to their role as they are usually salaried. However, bonuses for coordinators may improve productivity, recruitment and retention, at the same time it may negatively impact autonomy and trust of participants [3].

It has been reported that the response rate of health professionals to postal surveys was significantly increased by offering them monetary incentives. Thus, there is possibility of increasing participation in research study by offering financial incentives for family doctors [2]. There are other factors that influence the family doctors' decision to participate in biomedical research, such as their attraction to the research topic, the goals of the study, affiliation with academia, professional requirements, and medical care benefits for their institutions [2]. In general, financial incentives are not the major factor that affects the family doctor's decisions of participation in clinical research, although proper financial incentives is important to compensate for their time and effort. There is no valid argument to prove that reimbursing family doctors for recruiting subjects is not acceptable practice [2]. They get paid to be compensated for the extra time they spend explaining to subjects about the importance of the study. Actually, it may even improve the retention rates as the physician will have enough time to explain the study details, purpose, and requirements for the potential subject. However, to alleviate the concern of conflict of interest the physician can disclose the payment he is going to receive for the subjects during the informed consent process [2].

Others argue that most trials are sponsored and funded by pharmaceutical companies and their main goal is commercialization. Doctors who are going to recruit the subjects have little or no influence over the study hypothesis, design, protocol, methods, reporting, safety monitoring, or even the decision to publish the study outcomes [10]. These trials rely on the amount of payment given to clinicians as motivation to recruit subjects not on the importance of research itself. Well-designed trials by non-commercial sponsors with the aim to answer an important question that do not pay recruiters usually fail to attract doctors. Hence, a commercialized driven system that does not take into consideration the importance of subjects' altruism in clinical trials can be unethical and unacceptable [10]. "One American study found that just over half of patients questioned found payments to clinicians unacceptable. "An even greater proportion (80\%) believed that the patient had a right to know that their doctor would be paid for enrolling them" [10]. Fully informed consent should include all information and a frank disclosure of payments provided to physicians that recruits the future subjects [10]. Beside the monetary incentive, investigators must evaluate, identify and apply different strategies that enhance subject recruitment and retention and eliminate barriers that may hinder their participation in clinical trials [7]. For example, flexible hours for participation, provide transportation, choose a convenient place and time for recruitment, eliminate cultural barriers, use community consultation, allocate sufficient staffing and time for recruitment process [7].

\section{Vulnerable population}

Vulnerable population is a term that refers to the disadvantaged sub-segment of the general public requiring maximum care and particular special protections in research. Vulnerable population require close and careful attention during the clinical trial design with notable recruitment considerations and high quality observation methods of overall safety and efficacy strategies ensuing research [11]. The vulnerable populations include but not limited to children, minors, pregnant women, prisoners, employees, critically ill, unconscious, disabled individuals, elderly people, ethnic minorities, international research, and economically and educationally disadvantaged [11].

The IRBs are responsible to protect the rights, well-being, safety, and privacy of vulnerable subjects. The role of regulatory bodies is crucial in biomedical research and it becomes more critical when a study involves vulnerable population. A special attention should be given to informed consent process when dealing with vulnerable population. Researchers may require using audiovisual and illustrative tools to boost the quality of consent process [11]. For instance, to attain scientific evidence that provides diagnostic, preventive, and therapeutic data, the involvement of pregnant and lactating women in clinical research is important [11]. Research in intellectually disabled group and children is challenging and burdensome task for investigators because cognition of the subjects is essential for understanding and signing the informed consent thus surrogate consent is mandated [11].

As a result of the continuous attention on the rights, interests and well-being of research subjects, the need for community involvement in research development and approval has become apparent. Despite the concentration of the ethical guidelines on individuals, frequently, community consultation has become a common practice of involving communities [12]. Some argue the need for establishing ethical guidelines that require community disclosure, consultation, and consent. There should be clear ethical guidelines that guide the practice of community involvement in research [12].

\section{Conclusion}

When obtaining the informed consent from the subject or the legally guardian, the possibility of coercion or undue influence must be minimized [5]. It is important to adhere to the principles of GCP through the clinical research process to protect vulnerable population [11]. The need for examining and evaluating different methods for maximizing subject recruitment and retention is obvious. Since the role of research is critical for health care system advancement, there is a need for finding methods to provide incentives with minimizing conflict of interest and maximizing preferable outcomes [3] Thus, more research is required to set up ethical guidelines that regulate the incentive aspect in clinical research. Further research is needed to assess the appropriate payment method and amount for research subjects [9]. Undue inducement can be eliminated by careful assessment of risks, paying attention to eligibility criteria, collecting an informed and voluntary consent of research subjects [8]. A clear and factual guidance from regulatory bodies is important to lead researchers conducting research that involves vulnerable population. Vulnerable communities should be treated with concern, respects, equitably and in accordance with GCP principles [11].

I believe that adhering to GCP guidelines is a key element for conducting an ethical clinical research. However, more research is needed to help modeling those guidelines to maximize human subject recruitment process and protection. Also, I think that Community consultation is another crucial element that might foster the recruitment process as when you involve the community in research design and development this may foster their commitment for the success of the study. Their involvement helps them to understand the goals of the study thus may enhance the "buy in" attitude. 


\section{References}

1. DeRenzo EG, Moss J (2006) Writing clinical research protocols: Ethical considerations. Burlington, MA: Elsevier Academic Press.

2. Draper H, Wilson S, Flanagan S, Ives J (2009) Offering payments, reimbursement and incentives to patients and family doctors to encourage participation in research. Family Practice 26: 231-238

3. Dickert NW, Halpern SD, Butler J (2013) Incentivizing recruitment and retention to address enrollment challenges in clinical research. American Heart Association 1: $367-$ 370. [Crossref]

4. Vijayananthan A, Nawawi $\mathrm{O}$ (2008) The importance of good clinical practice guidelines and its role in clinical trials. Biomedical Imaging Intervention Journal 4: e5. [Crossref]

5. Smith-Tyler J (2007) Informed consent, confidentiality, and subject rights in clinical trials. Proceedings of the American Thoracic Society 4: 189-193. [Crossref]
6. Nardini C (2014) The ethics of clinical trials. E Cancer Medical Science 8: 387

7. Gul RB, Ali PA (2010) Clinical trials: the challenge of recruitment and retention of participants. Journal of Clinical Nursing 19: 227-233.

8. Grady C (2005) Payment of clinical research subjects. The Journal of Clinical Investigation 115: 1681-1687

9. Resnik DB (2015) Bioethical issues in providing financial incentives to research participants. Medicolegal and Bioethics 5: 35-41.

10. Rao JN, Cassia LJ (2002) Ethics of undisclosed payments to doctors recruiting patients in clinical trials. British Medical Journal 325: 36-37.

11. Shivayogi $\mathrm{P}$ (2013) Vulnerable population and methods for their safeguard. Perspectives in Clinical Research 4: 53-57.

12. Dickert N, Sugarman J (2005) Ethical goals of community consultation in research American Journal of Public Health 95: 1123-1127.

Copyright: $\odot 2018$ Ahmad W. This is an open-access article distributed under the terms of the Creative Commons Attribution License, which permits unrestricted use, distribution, and reproduction in any medium, provided the original author and source are credited. 